\title{
The convergence of a new symmetric iterative splitting method for non-autonomous systems
}

\author{
Gamze Tanoğlu* and Sila Korkut \\ Izmir Institute of Technology, Gulbahce Campus, Urla, Izmir 35430, Turkey
}

(Received 15 September 2011; revised version received 6 February 2012; accepted 3 April 2012 )

\begin{abstract}
The iterative splitting methods have been extensively applied to solve complicated systems of differential equations. In this process, we split the complex problem into several sub-problems, each of which can be solved sequentially. In this paper, we construct a new symmetric iterative splitting scheme based on the Magnus expansion for solving non-autonomous problems. We also study its convergence properties by using the concepts of stability, consistency, and order. Several numerical examples are illustrated to confirm the theoretical results by comparing frequently used methods.
\end{abstract}

Keywords: iterative scheme; non-autonomous system; convergence analysis; Magnus series

2010 AMS Subject Classifications: 65L20; 65L70; 65M12

\section{Introduction}

The main objective of this paper is to develop and analyse a splitting method for non-autonomous evaluation equation of the form

$$
\begin{aligned}
\frac{\mathrm{d}}{\mathrm{d} t} u(t) & =A(t) u(t), \quad t \geq 0, \\
u(0) & =u_{0} \in X
\end{aligned}
$$

on some Banach space $X$. The precise hypotheses on the operator family $A(t), 0 \leq t \leq t_{\text {end }}$, are given in Section 3. In order to solve such non-autonomous system, it is often the case that $A(t)=T+V(t)$, where only the potential operator $V(t)$ is time-dependent and $T$ is the differential operator $[1,3,9,10,15]$.

Operator splitting is a frequently used procedure in the numerical solution of large systems of partial differential equations. One of the operator splitting methods other than the classical Trotter and Strang splitting is the iterative splitting scheme which is based on first splitting the complex problem into simpler differential equations. Then, each sub-equation is combined with the iterative schemes, each of which is efficiently solved with suitable integrators [11-14]. Furthermore, these

\footnotetext{
*Corresponding author. Email:gamzetanoglu@iyte.edu.tr

ISSN 0020-7160 print/ISSN 1029-0265 online

(C) 2012 Taylor \& Francis

http://dx.doi.org/10.1080/00207160.2012.687447

http://www.tandfonline.com
} 
sub-equations can be considered as non-homogenous initial value problems (IVPs) which are also discussed in [7].

Some splitting methods have already been used to find numerical solution of the different special non-autonomous systems, particularly Hamiltonian ones $[5,8,18]$. It is important to develop such numerical schemes for Hamiltonian dynamics or Schrödinger equations that preserve some important qualitative properties and geometric structure of that solution. In this study, we focus on developing a new symmetric iterative scheme. We embed the Magnus expansion $[6,8]$ which is a popular geometric, an attractive and a widely applied method of solving explicitly time-dependent problems, in the solutions of the time-dependent split subsystem of the iterative scheme.

Our main focus is two-fold: first, we develop an iterative splitting for non-autonomous problem. Second, its convergence properties are analysed using the concepts of stability, consistency, and order as an abstract Cauchy problem via an analytic semigroup approach.

The plan of the paper is as follows: in Section 2, the basic idea behind the Magnus method is summarized. In Section 3, the algorithm of the symmetric iterative scheme is presented and its convergence properties are studied. In Section 4, several numerical examples are illustrated to confirm our theoretical results and efficiency of the new scheme.

\section{Exponential splitting method based on the Magnus expansion}

The Magnus integrator was introduced as a tool to solve non-autonomous linear differential equations for linear operators of the form

$$
\frac{\mathrm{d} u}{\mathrm{~d} t}=A(t) u(t)
$$

with solution

$$
u(t)=\exp (\Omega(t)) u(0) .
$$

The Magnus expansion is defined as

$$
\Omega(t)=\sum_{n=1}^{\infty} \Omega_{n}(t),
$$

where the first few terms are [8]

$$
\begin{aligned}
& \Omega_{1}(t)=\int_{0}^{t} \mathrm{~d} t_{1} A_{1}, \\
& \Omega_{2}(t)=\frac{1}{2} \int_{0}^{t} \mathrm{~d} t_{1} \int_{0}^{t_{1}} \mathrm{~d} t_{2}\left[A_{1}, A_{2}\right], \\
& \Omega_{3}(t)=\frac{1}{6} \int_{0}^{t} \mathrm{~d} t_{1} \int_{0}^{t_{1}} \mathrm{~d} t_{2} \int_{0}^{t_{2}} \mathrm{~d} t_{3}\left(\left[A_{1},\left[A_{2}, A_{3}\right]+\left[A_{3},\left[A_{1}, A_{2}\right]\right]\right),\right.
\end{aligned}
$$

where $A_{n}=A\left(t_{n}\right)$. In practice, it is more useful to define the $n$ th-order Magnus operator

$$
\Omega^{[n]}(t)=\Omega(t)+O\left(t^{n+1}\right)
$$

such that

$$
u(t)=\exp \left[\Omega^{[n]}(t)\right] u(0)+O\left(t^{n+1}\right) .
$$


For example, the second-order Magnus method is

$$
\Omega^{[1]}(t)=\int_{0}^{t} \mathrm{~d} t_{1} A\left(t_{1}\right)=\frac{t(A(t))+(A(0))}{2}+O\left(t^{3}\right) .
$$

Henceforth, the solution will be obtained as follows:

$$
u(t)=\mathrm{e}^{(t(A(t))+(A(0))) / 2} u(0)+O\left(t^{3}\right) .
$$

\section{Symmetric iterative splitting method and its convergence analysis}

\subsection{Derivation of the algorithm for symmetric iterative splitting}

Let us consider the IVP given in Equation (1) with the initial condition (2) on the time interval $\left[0, t_{\text {end }}\right]$, where $t_{\text {end }} \in R$. We suppose that $A(t)$ splits into the two parts as $T+V(t)$.

Let us divide the integration interval $\left[0, t_{\text {end }}\right]$ into $n$ equal parts by the points $t_{0}, t_{1}, \ldots, t_{n}$, where the length of each interval is $h=t_{j+1}-t_{j}=t_{\text {end }} / n, j=0,1 \ldots n$. The approximated solution and exact solution at time $t=t_{n}$ are $U\left(t_{n}\right)=U^{n}$ and $u\left(t_{n}\right)=u^{n}$, respectively.

We apply the second-order iterative process described as below on each subinterval $\left[t_{j}, t_{j+1}\right]$,

$$
\begin{aligned}
& \dot{u}_{1}=T u_{1}+V(t) U\left(t_{j}\right) \quad u_{1}\left(t_{j}\right)=U\left(t_{j}\right), \\
& \dot{u}_{2}=T u_{1}+V(t) u_{2} \quad u_{2}\left(t_{j}\right)=U\left(t_{j}\right),
\end{aligned}
$$

where $u_{2}\left(t_{j}\right)=U\left(t_{j}\right)$ denotes the numerical approximation to the true solution $u\left(t_{j}\right)$ at the time $t=t_{j}$ and $U\left(t_{0}\right)=u_{0}$. The formal solution of the sub-equations given in Equations (11) and (12) on the time interval $[t, t+h]$ can be written as

$$
u_{i}(t+h)=\Phi_{i}(t+h, t) U(t)+\int_{t}^{t+h} \Phi_{i}(t+h, s) F_{i}(s) \mathrm{d} s, \quad i=1,2,
$$

where $F_{1}=V(t) U(t), F_{2}=T u_{1}(t)$ and $\Phi_{1}(t+h, t)=\mathrm{e}^{h T}, \Phi_{2}(t+h, t)=\mathrm{e}^{(h / 2)[V(t+h)+V(t)]}$.

Next, we use the trapezoidal rule to approximate the integral

$$
\int_{t}^{t+h} \Phi_{i} F_{i} \mathrm{~d} s=\frac{h}{2}\left[F_{i}(t+h)+\Phi_{i}(t+h, t) F_{i}(t)\right]+O\left(h^{3}\right) .
$$

Note that $\Phi_{i}(t+h, t+h)=I$. After combining approximation (13) with the iterative schemes (11) and (12) and rearranging expressions, we obtain the first-order approximation

$$
u_{1}\left(t_{n}+h\right)=\mathrm{e}^{T h}\left[u_{1}\left(t_{n}\right)+\frac{h}{2} V\left(t_{n}\right) u_{0}\left(t_{n}\right)\right]+\frac{h}{2} V\left(t_{n}+h\right) u_{0}\left(t_{n}\right)
$$

and the second-order approximation

$$
u_{2}\left(t_{n}+h\right)=\mathrm{e}^{(h / 2)\left[V\left(t_{n}+h\right)+V\left(t_{n}\right)\right]}\left[U\left(t_{n}\right)+\frac{h}{2} T u_{1}\left(t_{n}\right)\right]+\frac{h}{2} T u_{1}\left(t_{n}+h\right),
$$

where $U\left(t_{n}+h\right)=u_{2}\left(t_{n}+h\right)$. Repeat this procedure by taking $u_{0}\left(t_{n}\right)=u_{2}\left(t_{n}+h\right)$ for next interval until the desired time $t_{\text {end }}$ is reached.

PROPOSITION 3.1 New iterative scheme preserves the time-symmetry property. 
Proof The time-symmetry preservation can be easily seen by interchanging $t_{n+1}, u_{i}\left(t_{n+1}\right), h$ by $t_{n}, u_{i}\left(t_{n}\right),-h$, respectively. In order to simplify the notation, we write $u_{i}^{n}$ instead of $u_{i}\left(t_{n}\right)$.

$$
u_{1}^{n}=\mathrm{e}^{-h T}\left[u_{1}^{n+1}-\frac{h}{2} V\left(t_{n+1}\right) u_{0}^{n}\right]-\frac{h}{2} V\left(t_{n}\right) u_{0}^{n} .
$$

By rearranging Equation (16), we have

$$
\begin{aligned}
\mathrm{e}^{h T}\left[u_{1}^{n}+\frac{h}{2} V\left(t_{n}\right) u_{0}^{n}\right] & =\left[u_{1}^{n+1}-\frac{h}{2} V\left(t_{n+1}\right) u_{0}^{n}\right] \\
u_{1}^{n+1} & =\mathrm{e}^{h T}\left[u_{1}^{n}+\frac{h}{2} V\left(t_{n}\right) u_{0}^{n}\right]+\frac{h}{2} V\left(t_{n+1}\right) u_{0}^{n} .
\end{aligned}
$$

Similarly, when we consider the second-order scheme to prove the time-symmetry property, we use the same procedure as above:

$$
u_{2}^{n}=\mathrm{e}^{-(h / 2)\left[V\left(t_{n}\right)+V\left(t_{n+1}\right)\right]}\left[u_{2}^{n+1}-\frac{h}{2} T u_{1}^{n+1}\right]-\frac{h}{2} T u_{1}^{n} .
$$

When we arrange Equation (17) for $u_{2}^{n+1}$, it is equivalent to equation in Equation (12).

We note that the number of iterations are restricted to two, since in order to compare our method with Lie-Trotter splitting method (first-order method) and Strang splitting method (second-order method).

\subsection{Convergence analysis}

In this section, we analyse the convergence behaviour of the new symmetric iterative scheme derived in the previous section. We assume that $T$ is an unbounded and $V(t)$ is a bounded operator. We define an operator norm as $\|\cdot\|_{X \leftarrow X}$ in a (complex) Banach space $\left(X,\|\cdot\|_{X \leftarrow X}\right)$.

In our proofs, we use the following hypotheses.

Hypothesis 1: Suppose that the closed linear operator $A(t): D \rightarrow X$, where $D$ is a dense subset of $X$, and that $A(t)$ is uniformly sectorial for $0 \leq t \leq t_{\text {end }}$. Then, there exist constants $a \in \mathbb{R}, 0<\varphi<\pi / 2$, and $M_{1} \geq 1$ such that $S_{\varphi}(a)=\{\lambda \in \bar{C}:|\arg (a-\lambda)| \leq \varphi\} \cup\{a\}$,

$$
\left\|(\lambda I-A(t))^{-1}\right\|_{X \leftarrow X} \leq \frac{M_{1}}{|(a-\lambda)|} \quad \text { for any } \lambda \in \mathbb{C} \backslash S_{\varphi}(a) .
$$

Then for fixed $0 \leq s \leq t_{\text {end }}$, the analytic semigroup $\mathrm{e}^{t A(s)}$ satisfies $\left\|\mathrm{e}^{t A(s)}\right\| \leq M \mathrm{e}^{\omega t}$ for some constants $\omega<0$ and $M \geq 1$. Our general references on semigroups are [2,16].

Hypothesis 2: Let $D(T)=D(A(t))$. We assume that $T$ is a linear closed operator and generates a strongly continuous semigroup $\mathrm{e}^{t T}$ on $\mathrm{X}$. By semigroup property, we assume $\left\|\mathrm{e}^{T t}\right\| \leq 1$.

Hypothesis 3: We assume that $V(t)$ is a bounded linear operator on some Banach space X. By means of Equation (10), we get $\mathrm{e}^{\Omega_{V}(t)} \leq \mathrm{e}^{t\|V(t)\|}$, where $\Omega_{V}(t) \approx \Omega_{1}(t)$, as the convergence of the Magnus expansion is guaranteed if $\|\Omega(t)\|<\pi$. The details can be found in [19].

Hypothesis 4: Let $T$ be an infinitesimal generator of a $\mathcal{C}_{0} \operatorname{semigroup} S(t), t \geq 0$. Let $t_{\text {end }}>0$. We have

$$
\sup _{0 \leq t \leq t_{\text {end }}}\|T u(t)\| \leq E_{1}\left(t_{\text {end }}\right), \quad i=0,1,2,
$$

where $E_{1}$ depends on the specific choice of $t_{\mathrm{end}}, T, V(t) u_{0}$, and $u_{0}$. For a detailed proof, see [4]. 
Hypothesis 5: We assume that there are non-negative constants $\tilde{C}$ and $R$ with

$$
\begin{gathered}
\sup _{0 \leq t \leq t_{\text {end }}}\|V(t)\| \leq \tilde{C}, \\
\|u\| \leq R \quad \text { on } \quad 0 \leq t \leq t_{\text {end }}
\end{gathered}
$$

Under these conditions, the following convergence analysis is obtained for the proposed symmetric iterative scheme.

PROPOSITION 3.2 The symmetric iterative splitting is of first order if we consider only one iteration given in Equation (11) with the error bound

$$
\|u(h)-U(h)\| \leq K h^{2} .
$$

Here, $K$ only depends on $\tilde{C}, R, E_{1}\left(t_{\mathrm{end}}\right)$.

Proof We define the local error by $e_{j}=U\left(t_{j}\right)-u\left(t_{j}\right), j=0,1,2, \ldots, n$. For simplicity, we only consider the time interval $[0, h]$. The exact solution of Equation (1) can be written as

$$
u(h)=\mathrm{e}^{T h} u_{0}+\int_{0}^{h} \mathrm{e}^{T h} V(h-s) u(h-s) \mathrm{d} s .
$$

We derive the error bound for Equation (1) by using the first-order iterative splitting scheme. Thus, the numerical solution of Equation (1)

$$
U(h)=\mathrm{e}^{T h} u_{0}+\int_{0}^{h} \mathrm{e}^{T h} V(h-s) u_{0} \mathrm{~d} s .
$$

By subtracting Equation (22) from Equation (21) leads to

$$
\begin{aligned}
\|u(h)-U(h)\| & =\left\|\int_{0}^{h} \mathrm{e}^{T h} V(h-s)\left[u(h-s)-u_{0}\right] \mathrm{d} s\right\| \\
& \leq h \tilde{C}\left\|u(h-s)-u_{0}\right\|,
\end{aligned}
$$

where $\tilde{C}=\sup _{0 \leq t \leq t_{\text {end }}}\|V(t)\|$. To obtain the error bound for $\left\|u(h)-u_{0}\right\|$, we use Hypotheses 4 and 5 ,

$$
\begin{aligned}
u(h) & =u_{0}+\int_{0}^{h} A(s) u(s) \mathrm{d} s, \\
\left\|u(h)-u_{0}\right\| & =h\|A(s) u\|=h\|(T+V(s)) u\| \leq h\|T u\|+\|V(s) u\|, \\
\left\|u(h)-u_{0}\right\| & \leq h\left(E_{1}\left(t_{\text {end }}\right)+\tilde{C} R\right) .
\end{aligned}
$$

By substituting Equation (24) in Equation (23), we have

$$
\|u(h)-U(h)\| \leq h^{2} \tilde{C}\left(E_{1}\left(t_{\mathrm{end}}\right)+\tilde{C} R\right)=K h^{2},
$$

where $K=\tilde{C}\left(E_{1}\left(t_{\text {end }}\right)+\tilde{C} R\right)$.

PROPOSITION 3.3 The symmetric iterative splitting is of second order if we consider two iterations given in Equation (12) with the error bound

$$
\|u(h)-U(h)\| \leq \tilde{K} h^{3} .
$$

Here, $\tilde{K}$ only depends on $\tilde{C}, R, E_{1}\left(t_{\text {end }}\right)$. 
Proof We write the equation for second-order iterative splitting as follows:

$$
U(h)=\mathrm{e}^{T h} u_{0}+\int_{0}^{h} \mathrm{e}^{T h} V(h-s) u_{1} \mathrm{~d} s .
$$

For estimating the error bound, we subtract Equation (27) from Equation (21), and the remaining term is

$$
\begin{aligned}
\|u(h)-U(h)\| & =\left\|\int_{0}^{h} \mathrm{e}^{T h} V(h-s)\left[u(h-s)-u_{1}\right] \mathrm{d} s\right\| \\
& \leq h \tilde{C}\left\|u(h-s)-u_{1}\right\| .
\end{aligned}
$$

Here, $u_{1}$ is the solution of the equation in Equation (11). The proof follows that of the bound of $\left\|u(h-s)-u_{1}\right\|$ in Equation (25), and we have

$$
\|u(h)-U(h)\| \leq h^{3} \tilde{C}^{2}\left(E_{1}\left(t_{\mathrm{end}}\right)+\tilde{C} R\right)=h^{3} \tilde{K} .
$$

PROPOSITION 3.4 The symmetric second-order iterative splitting scheme is stable on $\left[0, t_{\mathrm{end}}\right]$ with the bound

$$
\left\|U^{n}\right\| \leq \mathrm{e}^{t_{\text {end }} \tilde{C}}\left\|u_{0}\right\|+h \mathrm{e}^{2 h \tilde{C}} E_{1}\left(t_{\mathrm{end}}\right)\left(\frac{1-\mathrm{e}^{t_{\mathrm{end}} \tilde{C}}}{1-\mathrm{e}^{h \tilde{C}}}\right) .
$$

Proof For proving the above stability bounds, we employ the standard techniques. For the purpose, we start with the needed auxiliary stability bound of the first-order iterative splitting as in the following proof:

$$
U^{1}=U(h)=\mathrm{e}^{T h} U^{0}+F_{1}, \quad U^{0}=u_{0},
$$

where

$$
F_{1}=\int_{0}^{h} \mathrm{e}^{T h} V(h-s) u_{0},
$$

which is bounded by $\left\|F_{1}\right\| \leq h \tilde{C}\left\|u_{0}\right\|$, where $\tilde{C}=\sup _{0 \leq t \leq t_{\text {end }}}\|V(t)\|$. By rearranging Equation (30)

$$
\begin{aligned}
\left\|U^{1}\right\| & =\left\|\mathrm{e}^{T h} U^{0}+F_{1}\right\|, \\
\left\|U^{1}\right\| & \leq\left\|\mathrm{e}^{T h} U^{0}\right\|+\left\|F_{1}\right\|, \\
\left\|U^{1}\right\| & \leq\left\|u_{0}\right\|+\left\|h \tilde{C} u_{0}\right\|=(1+h \tilde{C})\left\|u_{0}\right\| .
\end{aligned}
$$

Recursively, we get the stability polynomial for the iterative scheme at first order

$$
\left\|U^{n}\right\| \leq(1+h \tilde{C})^{n}\left\|u_{0}\right\|=\mathrm{e}^{t_{\text {end }}} \tilde{C}\left\|u_{0}\right\| .
$$

On the other hand, for finding the stability result of the second-order iterative splitting, we use closeness and linearity of $T$. It follows as

$$
U^{1}=\mathrm{e}^{\Omega_{V}(h)} U^{0}+F_{2},
$$

where

$$
F_{2}=\int_{0}^{h} \Phi_{2}(t, s) T u_{1},
$$

which is bounded by $\left\|F_{2}\right\| \leq h \mathrm{e}^{2 h \tilde{C}}\left\|T u_{1}\right\|$. Since $T$ is a closed operator and for all $i=1,2 \ldots n$, by using Hypothesis $4,\|T U\| \leq E_{1}\left(t_{\text {end }}\right)$. Substituting the bound of $F_{2}$ into Equation (33) leads 
to

$$
\begin{aligned}
\left\|U^{1}\right\| & \leq\left\|\mathrm{e}^{\Omega_{V}(h)} U^{0}\right\|+\left\|F_{2}\right\|, \\
\left\|U^{1}\right\| & \leq\left\|\mathrm{e}^{h((V(h)+V(0)) / 2)} U^{0}\right\|+h \mathrm{e}^{2 h \tilde{C}} E_{1}\left(t_{\text {end }}\right), \\
\left\|U^{1}\right\| & \leq\left\|\mathrm{e}^{h \tilde{C}} U^{0}\right\|+h \mathrm{e}^{2 h \tilde{C}} E_{1}\left(t_{\text {end }}\right), \\
\left\|U^{1}\right\| & \leq\left\|\mathrm{e}^{h \tilde{C}} U^{0}\right\|+h \hat{H},
\end{aligned}
$$

where $\hat{H}=\mathrm{e}^{2 h \tilde{C}} E_{1}\left(t_{\text {end }}\right)$.

Recursively,

$$
\begin{aligned}
\left\|U^{1}\right\| & \leq\left\|\mathrm{e}^{h \tilde{C}} U^{0}\right\|+h \hat{H}, \\
\left\|U^{n}\right\| & \leq \mathrm{e}^{n h \tilde{C}}\left\|u_{0}\right\|+h \hat{H}\left[1+\mathrm{e}^{h \tilde{C}}+\cdots+\mathrm{e}^{h(n-1) \tilde{C}}\right], \\
\left\|U^{n}\right\| & \leq \mathrm{e}^{n h \tilde{C}}\left\|u_{0}\right\|+h \hat{H} \sum_{i=0}^{n-1} \mathrm{e}^{i h \tilde{C}}, \\
\left\|U^{n}\right\| & \leq \mathrm{e}^{t_{\text {end }} \tilde{C}}\left\|u_{0}\right\|+h \hat{H}\left(\frac{1-\mathrm{e}^{t_{\text {end }} \tilde{C}}}{1-\mathrm{e}^{h \tilde{C}}}\right), \\
\left\|U^{n}\right\| & \leq \mathrm{e}^{t_{\text {end }} \tilde{C}}\left\|u_{0}\right\|+h \hat{S},
\end{aligned}
$$

where

$$
\hat{S}=\mathrm{e}^{2 h \tilde{C}_{1}} E_{1}\left(t_{\mathrm{end}}\right)\left(\frac{1-\mathrm{e}^{t_{\mathrm{end}} \tilde{C}}}{1-\mathrm{e}^{h \tilde{C}}}\right) \quad \text { and } \quad \tilde{C}=\sup _{0 \leq t \leq t_{\mathrm{end}}}\|V(t)\| .
$$

PROPOSITION 3.5 The global error of iterative splitting is bounded by

$$
\left\|U^{n}(h)-u^{n}(h)\right\| \leq G h^{2} .
$$

Here, $G$ only depends on $t_{\mathrm{end}},\left\|u_{0}\right\|, R$, and $\tilde{C}$.

Proof We use the following insignificant modification of theorem in [17]. We can show by induction that the error after $n>0$ steps is

$$
U^{n}(h)-u^{n}(h)=\sum_{i=0}^{n-1} U_{h}^{i}\left(U_{h}-u_{h}\right) \mathrm{e}^{(n-1-i) h(T+V(t))} u_{0},
$$

where $U_{h}$ is the second order symmetric iterative scheme. Since $\left\|U_{h}^{i}\right\| \leq\left\|\mathrm{e}^{t_{\text {end }}} \tilde{C}_{u_{0}}+h \hat{S}\right\|$ and $\mathrm{e}^{(n-1-i) h(T+V(t))} u_{0}=u\left(t_{n-1-i}\right)$, this yields

$$
\begin{aligned}
\left\|U^{n}(h)-u^{n}(h)\right\| & \leq \sum_{i=0}^{n-1}\left\|\mathrm{e}^{t_{\text {end }} \tilde{C}}\right\| u_{0}\|+h \hat{S}\|\left\|\left(U_{h}-u_{h}\right) u_{n-1-i}\right\| \\
& \leq\left(\mathrm{e}^{t_{\text {end }}} \tilde{C}_{\| u_{0}}\|+\| h \hat{S} \|\right) \sum_{i=0}^{n-1}\left\|U_{h}-u_{h}^{n}\right\|\left\|u_{n-1-i}\right\|,
\end{aligned}
$$

and it follows from Proposition 3.4 that

$$
\left\|U^{n}(h)-u^{n}(h)\right\| \leq h^{2} G+\mathcal{O}\left(h^{3}\right),
$$

where $G=t_{\text {end }}\left\|u\left(t_{n-1-i}\right)\right\| \mathrm{e}^{t_{\text {end }} \tilde{C}}\left\|u_{0}\right\|$. 


\section{Numerical examples}

We first deal with the Mathieu equation

$$
q^{\prime \prime}+\left(\omega^{2}-\varepsilon \cos (t)\right) q=0 .
$$

This equation is solved by different techniques in [20]. By redefining the variables as $q(t)=q_{1}(t)$ and $\dot{q}(t)=q_{2}(t)$, and $u(t)=\left(q_{1}(t), q_{2}(t)\right)$, then the time-dependent oscillator corresponds to

$$
A(t)=\left(\begin{array}{cc}
0 & 1 \\
-\left(\omega^{2}-\varepsilon \cos t\right) & 0
\end{array}\right)=\left(\begin{array}{cc}
0 & 1 \\
-\omega^{2} & 0
\end{array}\right)+\left(\begin{array}{cc}
0 & 0 \\
\varepsilon \cos t & 0
\end{array}\right) \equiv T+V(t) .
$$

We take as initial condition $q(0)=1.75$ and $\dot{q}(0)=0$, integrate up to $t=10$, and measure the average error for different time steps.

The numerically observed order in the discrete $L^{\infty}$ norm is approximately 1 in Table 1 which is supported by Proposition 3.2. In addition, Proposition 3.3 predicts order 2. This number is in perfect agreement with Table 2 . We can also observe in Table 2 that the second-order symmetric iterative splitting scheme is more efficient than not only Strang splitting but also symmetrically weighted splitting (SWS) and the second-order Magnus method $\left(\Omega^{[1]}\right)$. We note that throughout this section, $\mathrm{SISM}_{i}$ denotes $i$ th-order symmetric iterative splitting.

Another example is the time-dependent Schrödinger equation as in the following form:

$$
i \hbar \frac{\partial \psi(x, t)}{\partial t}=\hat{H} \psi(x, t)
$$

where $\psi(x, t)$ denotes the probability amplitude for the particle to be found at position $x$ at time $t$ and $\hat{H}$ is the Hamiltonian operator for a single particle in a potential.

In our study, we choose a one-dimensional harmonic oscillator in the finite time interval $t \in$ $\left[0, t_{\mathrm{end}}\right]$ which has the form

$$
\begin{aligned}
i \frac{\partial \psi(x, t)}{\partial t} & =\left(-\frac{1}{2} \frac{\partial^{2}}{\partial x^{2}}+\frac{\omega^{2}(t)\left(x^{2}-1\right)}{2}\right) \psi(x, t), \\
\psi_{0}(x) & =\sqrt[4]{\frac{1}{\pi}} \exp \left(-\frac{1}{2} x^{2}\right)
\end{aligned}
$$

with $\omega^{2}(t)=4-3 e^{-t}$.

Table 1. Comparison of errors for several $h$ on $[0,10]$ interval with various methods, where $\omega=0.6$ and $\varepsilon=0.3$.

\begin{tabular}{lcc}
\hline$h$ & SISM $_{1} /$ order & Lie-Trotter/order \\
\hline 0.1 & 0.0610 & 0.1015 \\
0.001 & $6.6819 \mathrm{e}-004(0.9946)$ & $0.0011(0.9798)$ \\
\hline
\end{tabular}

Note: The expected order is 1 .

Table 2. Comparison of errors for different $h$ on $[0,10]$ interval with several methods, where $\omega=0.6$ and $\varepsilon=0.3$.

\begin{tabular}{lcccc}
\hline$h$ & SISM $_{2} /$ order & Strang splitting/order & SWS/order & $\Omega^{[1]}$ \\
\hline 0.1 & $9.8067 \mathrm{e}-004$ & 0.0011 & 0.0062 & 0.0014 \\
0.01 & $8.3542 \mathrm{e}-006(2.0696)$ & $1.0839 \mathrm{e}-005(2.0064)$ & $6.3187 \mathrm{e}-005(1.9918)$ & $1.3091 \mathrm{e}-005(2.0292)$ \\
0.001 & $8.2197 \mathrm{e}-008(2.0070)$ & $1.0801 \mathrm{e}-007(2.7672)$ & $6.3309 \mathrm{e}-007(1.9992)$ & $1.3009 \mathrm{e}-007(2.0027)$ \\
\hline
\end{tabular}

Note: Accepted exact solution is the fourth-order Magnus expansion. The expected order is 2. 

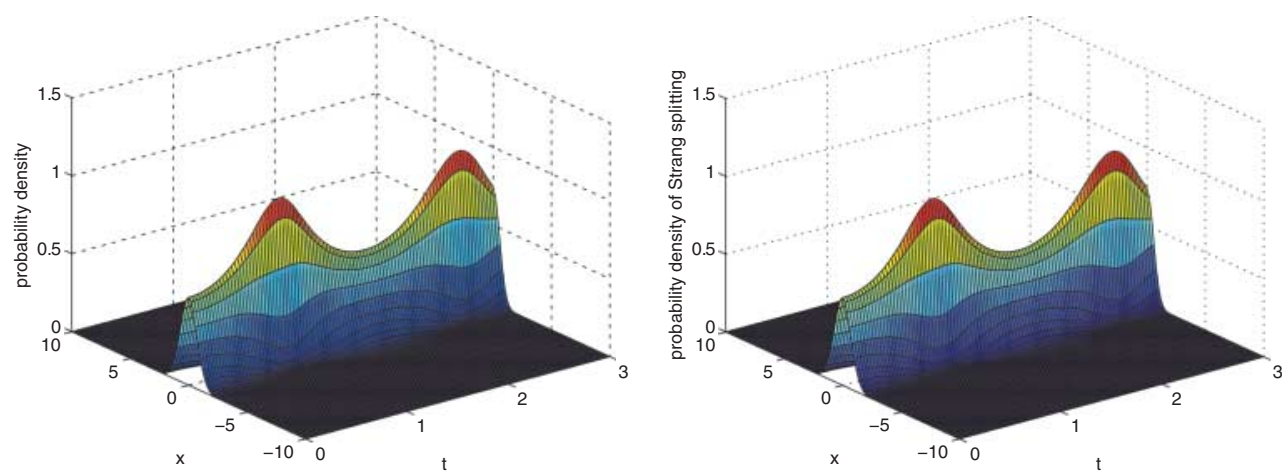

Figure 1. The probability of density, $|\Psi(x, t)|^{2}$, for the equation in Equation (40) by using SISM $_{2}$ on the left and by using the Strang splitting method on the right.

We take into account the system as in the following form:

$$
\left(\begin{array}{l}
\dot{u} \\
\dot{v}
\end{array}\right)=\left(\begin{array}{cc}
0 & A(t, x) \\
-A(t, x) & 0
\end{array}\right)\left(\begin{array}{l}
u \\
v
\end{array}\right),
$$

where $\psi(x, t)=u(x, t)+i v(x, t) . T$ corresponds to spatial derivative $\partial^{2} \psi(x, t) / \partial x^{2}$, we use the second-order centre difference scheme in order to approximate it, thus we get $2 N \times 2 N$ system.

$$
\left(\begin{array}{l}
\dot{u} \\
\dot{v}
\end{array}\right)=\left(\begin{array}{cc}
0 & T \\
-T & 0
\end{array}\right)\left(\begin{array}{l}
u \\
v
\end{array}\right)+\left(\begin{array}{cc}
0 & V(t, x) \\
-V(t, x) & 0
\end{array}\right)\left(\begin{array}{l}
u \\
v
\end{array}\right) .
$$

For exhibited Figure 1, we suppose that the system is defined in the interval $x \in[-10,10]$, which is split into $M=100$ parts of length $\Delta x=0.2$. We integrate the system using the proposed method with the time step size $\Delta t=0.03$ up to final time $t=3$. We observed that both methods preserve the probability density of the particle in Equation (40). We can choose our new method instead of the Strang splitting method, since we use two steps in $\mathrm{SISM}_{2}$, but three steps in Strang splitting method.

\section{Conclusions and discussions}

We have constructed a new symmetric iterative splitting scheme for non-autonomous systems with the help of the Magnus expansion. This new scheme is applicable for obtaining the numerical solution of the non-autonomous systems, for example, a Schrödinger equation in quantum mechanics, since it preserves the time symmetry. We also investigated the convergence properties of the new scheme by using the semigroup approaches. We confirm the theoretical results on a test problem. The method also provides the higher order accuracy in the approximate solution with an increasing number of iteration steps. Finally, numerical experiments reveal that our proposed method is efficient and easily adapted to numerically solve for such problems.

\section{References}

[1] V.C. Aguilera-Navarro, G.A. Estévez, and R. Guardiola, Variational and perturbative schemes for a spiked harmonic oscillator, J. Math. Phys. 31 (1990), pp. 99-104.

[2] A. Bátkai, P. Csomós, B. Farkas, and G. Nickel, Operator splitting for non-autonomous evolution equations, J. Funct. Anal. 260(7) (2011), pp. 2163-2190. 
[3] D. Baye, G. Goldstein, and P. Capel, Fourth-order factorization of the evolution operator for time-dependent potentials, Phys. Lett. A 317 (2003), pp. 337-342.

[4] M. Bjørhus, Operator splitting for abstract Cauchy problems, IMA J. Numer. Anal. 18 (1998), pp. 419-443.

[5] S. Blanes and P.C. Moan, Splitting methods for non-autonomous Hamiltonian equations, J. Comput. Phys. 170 (2001), pp. 205-230.

[6] S. Blanes and P.C. Moan, Fourth- and sixth-order commutator free Magnus integrators for, linear and nonlinear dynamical systems, Appl. Numer. Math. 56 (2006), pp. 1519-1537.

[7] S. Blanes and E. Ponsoda, Time-averaging and exponential integrators for non-homogeneous linear IVPs and BVPs, Appl. Numer. Math. 62(8) (2012), pp. 875-894.

[8] S. Blanes, F. Casas, J.A. Oteo, and J. Ros, The Magnus expansion and some of its applications, Phys. Rep. 470 (2009), pp. 151-238.

[9] S.A. Chin and P. Anisimov, Gradient symplectic algorithms for solving the radial Schrödinger equation, J. Chem. Phys. 124 (2006).

[10] S.A. Chin and C.R. Chen, Gradient symplectic algorithms for solving the Schrödinger equation with time-dependent potentials, J. Chem. Phys. 117(4) (2002), pp. 1409-1415.

[11] I. Farago, A modified iterated operator splitting method, Appl. Math. Model. 32(8) (2008), pp. 1542-1551.

[12] I. Farago and J.Geiser, Iterative operator-splitting methods for linear problems, Int. J. Comput. Sci. Eng. 3 (2007), pp. 255-263.

[13] J. Geiser, Decomposition Methods for Differential Equations: Theory and Application, CRC Press, London, May, 2009.

[14] J. Geiser, Iterative operator-splitting methods with higher-order time integration methods and applications for parabolic partial differential equations, J. Comput. Appl. Math. 217 (2008), pp. 227-242.

[15] G. Goldstein and D. Baye, Sixth-order factorization of the evolution operator for time-dependent potentials, Phys. Rev. E 70 (2004).

[16] C. González, A. Ostermann, and M. Thalhammer, A second-order Magnus-type integrator for nonautonomous parabolic problems, J. Comput. Appl. Math. 189 (2006), pp. 142-156.

[17] T. Jahnke and D. Altıntan, Efficient simulation of discrete stochastic reaction system with a splitting method, BIT Numer. Math. 50(4) (2004), pp. 797-822.

[18] L. Lara, A numerical method for solving a system of nonautonomous linear ordinary differential equations, Appl. Math. Comput. 170 (2005), pp. 86-94.

[19] C. Moan and J. Niesen, Convergence of the Magnus series, Found. Comput. Math. 8 (2008), pp. 291-301.

[20] J. Vigo-Aguiar and H. Ramos, Dissipative Chebyshev exponential-fitted methods for numerical solutions of secondorder differential equations, J. Comput. Appl. Math. 158 (2003), pp. 187-211. 